Projets

de paysage

\section{Projets de paysage}

Revue scientifique sur la conception et l'aménagement de l'espace

\section{$24 \mid 2021$}

Le paysage au prisme du politique

\title{
Le méthaniseur et le paysage
}

Le biodivers dans les situations problématiques d'aménagement du territoire

The Biogas Plant and the Landscape - Biodiversity in Problematic Land Use

Planning Situations

Fabrice Raffin et Camille Dormoy

\section{OpenEdition}

Journals

Édition électronique

URL : https://journals.openedition.org/paysage/20723

DOI : 10.4000 /paysage.20723

ISSN : 1969-6124

Éditeur :

École nationale supérieure du paysage de Versailles-Marseille, Institut national des sciences appliquées Centre Val de Loire - École de la nature et du paysage, École nationale supérieure d'architecture et de paysage de Bordeaux, École nationale supérieure d'architecture et de paysage de Lille, Agrocampus Angers

\section{Référence électronique}

Fabrice Raffin et Camille Dormoy, «Le méthaniseur et le paysage », Projets de paysage [En ligne], 24 | 2021, mis en ligne le 20 septembre 2021, consulté le 01 octobre 2021. URL : http:// journals.openedition.org/paysage/20723 ; DOI : https://doi.org/10.4000/paysage.20723

Ce document a été généré automatiquement le 1 octobre 2021.

\section{(c) (1) $\odot$}

La revue Projets de paysage est mise à disposition selon les termes de la Licence Creative Commons Attribution - Pas d'Utilisation Commerciale - Pas de Modification 4.0 International. 


\title{
Le méthaniseur et le paysage
}

\author{
Le biodivers dans les situations problématiques d'aménagement du \\ territoire
}

\author{
The Biogas Plant and the Landscape - Biodiversity in Problematic Land Use \\ Planning Situations
}

Fabrice Raffin et Camille Dormoy

\begin{abstract}
«Le mercredi 23 septembre 2020, ils sont un peu plus d'une centaine dans la cour de l'école de cette commune de mille habitants, située à une soixantaine de kilomètres de Paris. Sous un barnum blanc installé par la mairie, ils écoutent le chargé de mission de la coopérative qui accompagne le projet de méthanisation leur expliquer les caractéristiques de l'usine qui doit s'installer dans un champ, à 1,5 kilomètre des premières habitations, le long de la nationale. La présentation est interrompue plusieurs fois par l'impatience de certains participants, mais parvient à son terme. S'ensuivront plus de 3 heures d'échanges houleux relevant plutôt de l'invective, du "dialogue de sourds", voire de l'intimidation que du débat démocratique.» (Compte rendu d'observation de Fabrice Raffin de la réunion publique du 23 septembre 2020, organisée dans la cour de l'école de la commune.)
\end{abstract}

1 L'opposition des élus locaux et des populations à l'implantation d'usine de production de gaz méthane à partir de la fermentation de matières organiques (appelées ici unités ou usines de méthanisation) est aujourd'hui fréquemment observée (Bourdin et al., 2019 ; Kortsch et al., 2015). En se référant à l'un de ces projets d'implantation dans une petite commune située à une soixantaine de kilomètres de Paris, ce texte propose d'aborder «de l'intérieur» certains aspects de ces oppositions, en insistant sur la constitution de formes d'argumentation politique des protagonistes.

2 Pour ce faire, cet article se fonde sur une méthodologie participative que nous avons construite durant plus d'un an en tant que membre d'un cabinet d'études. Ce dernier avait été sollicité afin de réaliser un double diagnostic territorial pour l'implantation d'une unité de méthanisation par la coopérative agricole qui accompagne techniquement les agriculteurs. Au-delà de la réalisation des diagnostics eux-mêmes, nous avons rapidement acquis un rôle en tant que conseil pour répondre aux arguments des divers opposants au projet. C'est ainsi que nous avons pu participer à de nombreuses réunions de suivi du dossier tout au long de l'année, notamment des 
réunions imprévues liées à l'opposition au projet. Une opposition qui a débuté dans les services environnementaux de la DREAL qui ont provisoirement refusé de valider le projet et qui s'est poursuivie par la mobilisation des élus locaux (commune et communauté de communes), puis des habitants, jusqu'à une réunion publique organisée en extérieur pour raison de Covid. Nous avons également participé à d'autres types de réunions, organisées en préfecture ou à la communauté de communes ainsi qu'à des réunions techniques. L'ensemble constitue plus d'une vingtaine d'heures d'enregistrement, durant lesquelles nous étions identifiés comme membres du bureau d'études ayant des compétences en sciences humaines, mais pas en tant que chercheurs membres d'un laboratoire universitaire. C'est pourquoi à partir de ce rôle, nous pouvons parler d'observation participante et non de participation observante comme dans la plupart des recherches ethnographiques (Soulé, 2007). Nous avons, de plus, complété ces données par des entretiens avec certains protagonistes, une analyse de leur positionnement sur Internet, notamment sur les sites de référence des opposants aux projets de méthanisation et une veille concernant le traitement médiatique des unités de méthanisation dans la région.

C'est à partir de cette position que nous avons été amenés à analyser les oppositions que nous considérerons ici comme relevant du politique, mais également de la politique et des politiques du fait des décisions auxquelles elles conduisent, en tant que mise en œuvre d'orientations d'élus (municipalités, collectivités territoriales, État, citoyens). Simultanément, les oppositions mobilisent le politique comme conception/gestion collective de différentes thématiques (aménagement du territoire, économie, écologie, etc.), mais également en tant que conception de sa mise en œuvre et donc conception de l'action publique, la politique.

Ces situations d'opposition aux projets de méthanisation sont quasi systématiques aujourd'hui en France. Nous voudrions donc les considérer ici comme des situations problématiques de l'aménagement (Cefaï, 2013, et d'abord nous arrêter sur l'omniprésence des registres environnementaux ${ }^{1}$ dans la structuration des débats publics qu'elles génèrent. Plus précisément, il sera intéressant de montrer combien cette thématique environnementale est montée en généralité pour devenir un schème cohérent d'appréhension globale de la situation d'aménagement, sur fond de considérations locales du paysage. Puis, pointant comment ce registre se construit à travers un prisme systématiquement anthropomorphique ou humano-référencé, nous verrons, par ailleurs, combien la mobilisation de la science s'effectue à des fins d'argumentation, pour intervenir dans la constitution des publics (ibid.) de ces situations problématiques. Enfin, il s'agira simultanément, tout au long du texte, de montrer combien les références environnementales et scientifiques disent implicitement une part de représentations du paysage et participent en creux à sa "structuration négative " en empêchant les projets d'aménagement (en l'occurrence les unités de méthanisation) de voir le jour.

\section{Un dispositif sociotechnique d'envergure qui devient un problème}

«Le trouble rompt les habitudes de vie, le cours des choses ou la quiétude des mœurs, sans être encore transformé en objet de réflexion [...]. Lorsqu'une situation ordinaire traverse une telle crise, ses participants sortent de leur régime de conduite habituel et élaborent une expérience réflexive. Ils circonscrivent les 
éléments qui les troublent, s'interrogent sur leurs causes et leurs effets, s'enquièrent de leurs attitudes respectives vis-à-vis du trouble et examinent la signification sociale de leurs actes à cet égard. Ils discutent, ils raisonnent, ils s'inquiètent à haute voix, ils s'informent, ils enquêtent, ils avertissent l'opinion. Le trouble de l'expérience est converti en problème. » (Céfaï, 2013.)

5 Le processus d'implantation du dispositif sociotechnique que représente une unité de méthanisation mobilise un acteur-réseau (Callon et Law, 1997) extrêmement vaste sur une échelle spatio-temporelle allant du quotidien-local pour les villages concernés, au national, le tout, potentiellement, après démarrage de l'exploitation pour une durée minimale de $30 \mathrm{ans}^{2}$. Pour les projets qui aboutissent à la construction d'une unité de production, il faut près de trois ans entre la phase de conception et le début de l'exploitation. Pour le cas qui nous préoccupe, le collectif, constitué de 12 agriculteurs, accompagné par la coopérative agricole, a commencé son projet au printemps 2019.

6 Projet local, on pourra insister sur le fait que l'échelle spatio-temporelle de ces projets est en fait bien plus vaste, mobilisant le monde dans sa globalité. L'analyse des discours (réunions publiques, entretiens, sites Internet, presse locale) des opposants au projet montre ainsi combien les arguments environnementaux réfèrent en fait systématiquement au globe terrestre et aux problématiques écologistes mondialisées. Par exemple, la question d'un marais situé à 2 kilomètres du site d'installation de l'unité de méthanisation est rapprochée de la question de la rareté de l'eau dans le monde, voire de la pollution des mers ; le fait que ce soient des agriculteurs qui pilotent le projet conduit les opposants à l'associer à la question de l'agriculture capitaliste productiviste et, simultanément, à la question des pesticides et de la pollution des sols, de leur appauvrissement en général ; l'approvisionnement du site renvoie à la question de l'automobile et du transport par camion dans le monde ; la question du climat de la Terre est derrière chaque phrase et elle est ramenée à des enjeux quotidiens locaux. On pointera ainsi combien un processus d'aménagement local, en l'occurrence le méthaniseur, élément physique structurant pour un paysage situé, est lié aux débats environnementaux mondiaux sur le climat. Les argumentations dans le débat public réfèrent à un contexte (Abbott, 2016) marqué par l'abolition des échelles spatiotemporelles ou à leur nivellement, mettant sur un même plan des enjeux d'aménagement locaux et des enjeux environnementaux mondiaux comme le changement climatique. De plus, le contexte politique national participe d'une dramatisation de ces enjeux d'aménagement, en référence aux politiques environnementales gouvernementales. L'implantation d'une unité de méthanisation est vécue localement indirectement comme une imposition gouvernementale. Les élus locaux se sentent, sur ce point, dépossédés de leur territoire et in fine de leur paysage. Le paysage dans son ensemble, à l'échelle de la communauté de communes, est également considéré par les élus comme un atout de développement, pour le tourisme. Cette considération économique est partagée par les populations venues des villes de la périphérie parisienne. Elle s'accompagne d'une représentation d'un paysage naturaliste, bucolique et enchanté que les « rurbains » imaginent trouver et conserver dans un village à $60 \mathrm{~km}$ de Paris (Chesneau et Desplaces, 2018). Parmi nos interlocuteurs, la défiance que nous avons observée vis-à-vis de la préfecture, sensée être neutre mais considérée comme représentant l'État, est d'autant plus grande et révélatrice de ce point de vue 3 .

7 Enfin, un autre élément politique, de contexte local cette fois, joue également un rôle : des élus de la communauté de communes, notamment le nouveau maire du village, 
veulent montrer dans leur opposition au projet leur capacité à agir afin de renforcer leur légitimité politique, leur capacité à être aux côtés des habitants. Pourtant, lors d'échanges informels, certains élus locaux avaient exprimé que, initialement, ils n'étaient pas nécessairement opposés au projet. En toile de fond s'exprime ici non pas un attachement au paysage, mais plutôt une volonté de ne pas le changer, symbole aussi de la maîtrise du territoire. Mais ce contexte général influence aussi le positionnement du collectif d'agriculteurs qui porte le projet.

Figure 1. Schéma des acteurs

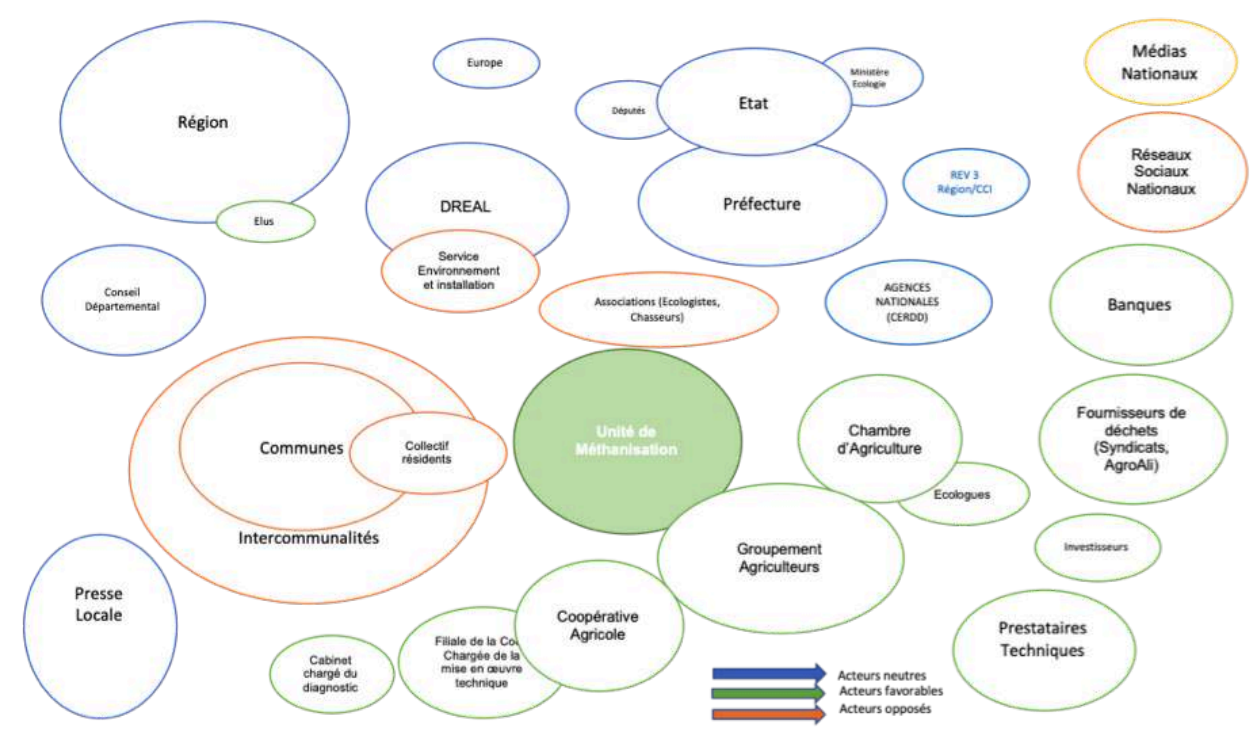

$8 \mathrm{Au}$ moment où nous commençons à participer aux réunions techniques et aux comités de pilotage de la coopérative, en février 2020, la démarche des agriculteurs est déjà entamée depuis près d'un an. L'énoncé du projet est lisible dans les échanges en réunion et dans le dossier constitué par les agriculteurs et la coopérative qui en est l'entité actrice (Callon et Law, 1997). Il présente les aspects techniques, les procédés et l'organisation du site, son raccordement au réseau de gaz, les dispositifs de stockage, les plans pour la construction, mais également la gouvernance du collectif, son modèle économique, la recherche de financements, côté banques et côté subventions publiques. Les douze agriculteurs sont âgés de 30 à 70 ans. Leurs exploitations sont très différentes en superficie comme en activités (maraîchers, céréaliers, éleveurs équins). Ils habitent dans les villages autour du site où doit être implanté le méthaniseur. Indépendamment de la taille de leur activité, ils ont choisi une gouvernance démocratique : partage en parts égales de l'investissement et, à terme, le partage aussi des éventuels bénéfices de l'activité.

Explicitement, la «philosophie » du projet se distingue d'autres projets industriels de grande envergure, financés par d'importantes entreprises du secteur de l'énergie. Parmi les agriculteurs, se trouve notamment un ingénieur agricole soucieux des enjeux écologiques. Il a largement participé à la conception technique du projet, traduisant sa perspective écologiste ${ }^{4}$ dans le dispositif technique. D'autres agriculteurs se sentent 
également très investis d'une responsabilité "environnementale", notamment le porte-parole du collectif qui, lui, a moins de 35 ans. Les autres membres sans être "militants» sont en phase avec ce positionnement. Le salarié de la coopérative qui accompagne les agriculteurs a lui-même intégré ce discours, le porte et l'argumente dans différentes instances. Ce point permet de comprendre que les choix techniques du projet tentent de répondre aux critiques adressées à la majorité des usines de méthanisation déjà en activité. Parmi les solutions proposées par le collectif, on notera les dimensions relativement modestes de l'usine (450 tonnes), la non-utilisation de cultures nourricières, l'utilisation exclusive de cultures secondaires ${ }^{5}$ non arrosées et de déchets ménagers locaux, le montage d'un circuit court pour leur acheminement, la couverture des stocks pour éviter les odeurs, la construction d'une chape étanche pour séparer l'usine du sol en cas d'inondation et surtout un process d'hygiénisation qui évite d'utiliser de l'eau et permet de recycler les déchets ménagers des collectivités locales. Le choix de cet ensemble de mesures très onéreuses se comprend par les positionnements individuels et la perspective « environnementaliste » du projet.

Pourtant, à l'instar des analyses de Daniel Cefaï, le projet d'installation est rapidement identifié par les élus et certains résidents comme un trouble. Et comme dans d'autres situations, on voit se constituer une argumentation contre le projet par la mobilisation de tiers (des experts, des scientifiques) et, sur cette base, des lignes d'opposition entre les «pour» et les «contre». Outre les peurs concernant différentes nuisances potentielles, celle que le paysage soit modifié et dénaturé revient à chaque réunion. Pourtant, le site n'a rien d'exceptionnel, il se trouve dans un champ de betteraves qui appartient à l'un des agriculteurs en bordure de national. Mais la question est posée dans plusieurs réunions: "est-ce que ça va se voir?", avec une réponse positive $a$ priori, et la sincérité des agriculteurs est attaquée : "Vous dites que vous allez prêter attention aux haies le long de l'usine, mais on sait très bien que vous allez planter trois arbres et que vous n'allez pas vous en occuper et puis ça va mettre plusieurs années à pousser » (extrait de la réunion dans une mairie d'un village de la communauté de communes concernée, janvier 2021). Les exemples (souvent malheureux) d'autres expériences de méthanisation sont ainsi mobilisés pour discréditer ceux qui n'ont que leurs paroles pour défendre leur projet. La crainte d'une « dénaturation » implicite du paysage est ainsi mobilisée contre le projet, dans un contexte de peur de l'urbanisation des franges métropolitaines.

11 Malgré les choix techniques qu'ils ont faits, les investissements financiers déjà engagés, la diversité de leurs profils socioéconomiques, les agriculteurs sont le plus souvent identifiés comme un groupe homogène, confondus fréquemment avec des représentants de l'agro-industrie sucrière, voulant " gagner plus d'argent ", méprisant l'environnement. Vision qui rend caduque la confiance dans leur «sincérité écologique ». L'accusation faite aux agriculteurs de n'avoir pas prévenu la mairie de leur projet « dans les temps » déclenchera la mobilisation des habitants, la création du «collectif anti-méthanisation» et des actions telles que des articles dans les médias locaux, la publication de tracts, la création d'une pétition, jusqu'à l'organisation de la réunion publique. 


\section{Le biodivers humanisé : quand les animaux se mêlent d'aménagement}

«Une action, sa préparation, son accomplissement, sa signification, ne résultent pas d'une simple projection de l'intention du sujet agissant mais sont répartis entre l'objet, l'acteur et l'environnement et se constituent au point de rencontre entre ces différents éléments. » (Madeleine Akrich, 1993.)

Le processus d'implantation du méthaniseur suit une procédure administrative stricte et mobilise des acteurs publics qui, pour beaucoup, sont des services de l'État. En l'occurrence, les premières réunions auxquelles nous assistons sont organisées dans le cadre d'un examen par la direction régionale de l'Environnement, de l'Aménagement et du Logement (DREAL) de l'impact environnemental du projet. Cet examen, dit « au cas par cas", est obligatoire pour les infrastructures susceptibles d'avoir un «effet négatif " sur l'environnement ${ }^{6}$. Il conduit ou non à une première autorisation de poursuivre la procédure d'implantation, avant de déposer une demande de permis de construire. Sont également présents dans ces réunions d'autres acteurs qui soutiennent les projets de méthanisation comme la chambre d'agriculture ou des écologues qui ont effectué le diagnostic environnemental.

Lors de la première de ces réunions que nous avons enregistrée, les agents de la DREAL ont souhaité des précisions sur la prise en compte des animaux présents sur le site par les porteurs de projet. La future usine, qui sera implantée le long d'une nationale devant prochainement passer en $2 \times 2$ voies, sera également située sur un futur " corridor écologique », autrement dit sur un site de passage pour les animaux.

«Le principal problème rencontré à la lecture du document (présenté par les agriculteurs) est le fait qu'on arrive à enclaver cette parcelle entre deux clôtures et la route, ce qui limite largement la fonctionnalité de cette parcelle pour le passage de la grande faune au final. » (Agent $\mathrm{n}^{\circ} 2$, service environnement, DREAL, avril 2020, enregistrement effectué le lendemain de la réunion lors d'un entretien avec les deux agents du service Environnement concerné.)

14 Face à cette critique formulée par les agents de la DREAL, les porteurs de projet ont accepté de revoir les plans de l'usine en élargissant le passage réservé aux animaux, proposant un traitement paysagé adapté à la faune et offrant un point d'eau. À la fin de la réunion, les représentants de la DREAL acceptaient ce plan. Le lendemain, les participants qui s'étaient quittés sur cet apparent compromis étaient néanmoins interpellés par la DREAL qui refusait finalement de valider le «cas par cas ». La proposition des agriculteurs n'était pas, pour la DREAL, suffisante pour le bien-être de la faune.

" On se retrouve dans une situation où on contraint énormément les animaux, c'est la grande faune qui est visée, qui est déjà très sensible à ce genre de phénomène, je ne suis pas convaincue en l'état actuel que ce soit suffisant [...], avec les aménagements, on va avoir une faune qui va devoir passer soit à droite soit à gauche, elle ne va pas pouvoir passer directement dessus, et avec les aménagements qui sont proposés, il y aura une haie et du coup ça va permettre de guider la faune qui ne va pas savoir par où passer. " (Agent $\mathrm{n}^{\circ} 2$, service environnement, DREAL, avril 2020, enregistrement effectué le lendemain de la réunion lors d'un entretien avec les deux agents du service environnement concerné.)

15 Au-delà de cet exemple et de manière récurrente, on soulignera ici l'humanisation des animaux en général et «du vivant » en particulier. Le jargon sociotechnique met en avant «la sensibilité » de la grande faune, mobilise des périphrases et dénonce les 
contraintes imposées aux animaux qui seront obligés de contourner le site du méthaniseur, alors qu'aujourd'hui, ils doivent traverser une route nationale sans aucun aménagement (sic). Les aménagements doivent, de plus, assurer «une fonctionnalité [...] pour la grande faune». L'intérêt des agriculteurs, et ce qu'ils apporteraient localement, n'est jamais mis en avant dans les débats. Au contraire, cet intérêt est plutôt évacué dans la mesure où ils sont soupçonnés d'être là uniquement pour " faire encore plus d'argent ». La négation des intérêts humains, des agriculteurs comme de la communauté locale, et la valorisation des intérêts des animaux par personnification caractérisent alors le traitement du dossier.

À ce stade, l'instruction administrative du "cas par cas " a montré des formes de militantisme inattendues dans un service de l'État. À bien regarder les profils de certains agents territoriaux, on le comprend d'autant mieux qu'ils sont à la fois fonctionnaires d'État, mais également militants associatifs, cumulant des rôles dans des associations de protection de l'environnement comme nous l'apprendrons plus tard.

Tout comme les frontières entre le local et le global tendent à s'effacer, celles entre mondes animal et humain sont aussi abolies faisant des animaux des acteurs du paysage, désormais pris en compte pour les aménagements, jusque dans l'aménagement des sites de production d'énergie. La faune comme élément de la biodiversité est élevée au rang d'acteur équivalant aux humains et, en ce sens, on pourra commencer à parler ici de la biodiversité humanisée, sous le mode de la personnification. Une biodiversité humanisée, c'est-à-dire la faune, la flore, le vivant, à qui, dans les discours, est prêtée une sensibilité, souvent une conscience, et par conséquent des droits. Une biodiversité humanisée que nous appellerons le biodivers, en une étape de réunification caractéristique de notre temps, pour ce qui concerne la distinction entre non-humain et quasi-humain depuis le XVII siècle comme l'a montré Bruno Latour (1991).

On notera ici combien indirectement, cette humanisation du biodivers influe sur la production du paysage. En effet, la prise en compte de ce qui est présenté comme le bien-être sensible des animaux influe directement sur les plans d'aménagement du site du méthaniseur. Les agriculteurs vont jusqu'à accepter non seulement de ménager un passage pour les animaux, mais également d'installer des mangeoires et des abreuvoirs, mais aussi de choisir les végétaux qui conviennent à leur alimentation dans les haies bordant le site. Comme si un "bon paysage " était un paysage où les animaux "se sentaient bien", selon la perspective anthropomorphique souvent appliquée à leur endroit dans les débats. Un paysage "monde des animaux », un paysage qui acquiert dès lors une valeur émotionnelle via l'attachement à la faune.

Malgré ces rebondissements, le «cas par cas» fut finalement validé par une double démarche entreprise par les agriculteurs dans les coulisses (Goffman, 1992) : d'un côté, fut produit un argumentaire étayé avec l'aide du cabinet d'experts concernant l'ensemble des enjeux écologiques du projet ; de l'autre, le député de la circonscription, élu de la majorité présidentielle, fut interpellé via les réseaux personnels du chargé de mission de la coopérative. Le parti au pouvoir soutenant explicitement les projets de méthanisation, il lui était demandé d'intervenir auprès de la DREAL en rappelant la position du gouvernement sur ces aménagements et le statut d'agents de l'État des protagonistes. 


\section{L'argumentation scientifique et la construction du biodivers} de l'humanisation dans le sens où sa construction et son omniprésence s'appuient en grande partie sur le discours scientifique.

«Lors de la réunion publique dans la cour de l'école, les agriculteurs ont fini de présenter leur projet de méthanisation alternative et le débat s'engage, tendu, critique, révélant une opposition majoritaire au projet. Le maire est présent, il ne prend la parole qu'une fois, en début de réunion. Une parole politique, militante et combative : bien qu'il ait peu parlé, c'est bien lui qui conduit l'opposition, assisté de deux autres élus moins rhétoriques, mais aux arguments plus scientifiques. Ils ont mobilisé les administrés via le conseil municipal d'abord, et par distribution de tracts dans les boîtes aux lettres ensuite. L'échange dure plus de 3 heures, il se termine par une fin de non-recevoir, des accusations de mensonges à l'endroit des agriculteurs, des menaces des opposants les plus remontés. Un fait a attiré mon attention à plusieurs reprises : les opposants ne connaissent pas la réalité du travail des agriculteurs, nombre de leurs arguments sont fondés sur une représentation objectivement erronée de leurs statuts divers et des techniques qu'ils emploient: les agriculteurs sont obligés d'expliquer qu'ils n'arrosent pas les cultures en été, sauf ceux, un seul sur les douze présents, qui sont irrigants. Par contre, leur argumentaire est implacable sur les dangers de la méthanisation, scientifiquement documentés.» (Compte rendu d'observation de la réunion publique du 23 septembre 2020.)

21 Ce n'est pas de la véracité des arguments mobilisés dont nous discuterons ici, mais de la manière dont ils sont mobilisés dans le débat, qui révèle un paradoxe. D’un côté, nous l'avons vu, une humanisation croissante de la biodiversité animalière et finalement la construction d'une continuité entre humains et non-humains, des liens continus avec l'environnement et le biodivers qui influe sur les plans d'aménagement d'un site de méthanisation. De l'autre, la mobilisation d'un discours scientifique objectivant, technique et froid, qui pointe les excès humains et qui réifie l'environnement, pour, paradoxalement, rétablir une frontière entre humains et non-humains.

Dans le débat public ce jour-là, les arguments de la traversée du site par la faune et des nuisances apportées aux animaux reviendront plusieurs fois. Mais il lui sera adjoint, de manière systématique, l'ensemble des arguments environnementaux récurrents des débats publics contemporains : l'eau, le climat et la question de l'énergie, les formes d'agricultures et la pollution de l'air et des sols, la santé des populations. Là encore, il n'y a pas de hiérarchisation des argumentations, mais uniquement une présentation de leur caractère exact et scientifique. Une référence à la science, appliquée de manière généralisée à tous les méthaniseurs, qui ne prend pas en compte le cas spécifique des agriculteurs qui ont tenté de répondre aux différentes critiques dont la méthanisation fait l'objet.

Pour construire ce discours, la place des réseaux sociaux est importante. Le site Internet français qui se présente comme le Collectif scientifique national méthanisation raisonnée (CNMR) fait référence pour les opposants à la méthanisation. Il propose des analyses scientifiques (chimiques, physiques, écologiques, etc.) pour tous les points environnementaux évoqués. Pourtant, à y regarder de plus près, le site propose des articles de revues reconnues mis au même niveau que les études construites par les animateurs du site et les avis dénonciateurs des opposants confrontés dans toute la 
France à des projets de méthanisation. Les niveaux d'argumentation se mêlent. La temporalité de la recherche se télescope avec le temps politique et l'urgence des oppositions locales, donnant un statut strictement politique aux arguments scientifiques, sans prendre en compte les réalités locales (enjeux de développement économiques, réalités du travail des agriculteurs). Il est par exemple frappant de voir des habitants argumenter, durant la réunion publique, de manière extrêmement technique, sur les échanges gazeux entre l'atmosphère et le sol, citant même des formules chimiques, mais ne sachant pas, nous l'avons dit, que la majorité des agriculteurs n'arrose pas leurs cultures. De plus, les contestataires prennent pour acquis des éléments encore en débat dans le monde de la recherche.

Cependant, il ne faudrait pas exagérer ce registre de défense de l'environnement. L'irruption systématique $d u$ biodivers dans le débat public n'indique pas nécessairement des postures militantes comme au niveau régional précédemment évoqué pour la DREAL. Durant notre réunion, un seul habitant opposé au projet, adjoint au maire, est apparu comme un véritable spécialiste des questions environnementales. Il était le principal passeur des thèses du site Internet du CNMR évoqué plus haut. Extrêmement "pointu» sur les questions écologiques et écologistes, il pouvait mobiliser des arguments de chimie, d'économie, de technologie, que les autres habitants reprenaient avec approximation. Le discours scientifique est bien alors partie prenante du débat et travaille donc le politique, mais les « convictions » le sont peutêtre moins.

Dans ce sens, le cas présent correspond également à une évolution des phénomènes Not In My BackYard (Nimby), abondamment analysés par les sciences sociales depuis les années 1980 (Béhar et Simoulin, 2014). L'opportunisme argumentaire côtoie une véritable préoccupation écologiste pour certains participants, mais pour beaucoup d'autres - la majorité des opposants que nous avons rencontrés - celle-ci reste plus diffuse que véritablement militante. De ce point de vue, nous relativiserons les analyses des phénomènes Nimby qui soulignent plutôt l'affaiblissement des intérêts particuliers, la prise de conscience des enjeux collectifs et le poids de l'argumentation désormais plus scientifique que passionnelle. En l'occurrence, derrière le discours scientifiquement argumenté de défense de l'environnement, au fil des réunions, la municipalité n'a eu de cesse de poser la question suivante : «Qu'est-ce que la commune y gagne? » D'un côté, un registre argumentaire écologique/écologiste; de l'autre, un registre argumentaire typique des Nimby sur l'intérêt local, qui écarte le premier et fait passer les arguments environnementaux au deuxième plan. Et durant la réunion publique, les habitants exposeront de manière récurrente leur peur de voir les prix du foncier baisser.

\section{Le paysage, la « fiction verte » et la pensée paysagère}

Des perceptions pacifiées de l'environnement irriguent et influencent le débat public. Des perceptions qui participent d'une attente vis-à-vis de l'action publique de l'aménagement : elle ne doit porter avec elle aucune nuisance pour quiconque, ni pour les habitants, ni pour la biodiversité.

Comme l'écrit Anaïs Belchun, et cela vaut pour le cas analysé ici, «la fiction verte tourne à plein régime [...], mais nous sommes pourtant bien loin des savoirs issus de l'écologie scientifique comme des concepts de l'écologie philosophique et culturelle » 
(Belchun, 2020). Les analyses scientifiques ne sont pas mobilisées pour penser la perspective et les effets écologiques des décisions d'aménagement. Précisément, il ne s'agit pas de s'intéresser réellement aux problématiques écologiques spécifiques posées par les choix techniques des agriculteurs. Ces derniers sont balayés a priori par les opposants, partant de l'idée que toute nouvelle implantation de dispositif pour produire de l'énergie aura des conséquences négatives, y compris sur le paysage, et qu'elle ne doit pas advenir. Ici se révèle à nouveau cet idéal que l'humain devrait avoir une action neutre envers ce qui est perçu comme "la nature", "respectant les animaux ", " avec un bilan carbone neutre ", "sans empreinte écologique » selon des termes que nous avons pu entendre dans les échanges avec et entre habitants.

Et de fait, cet argumentaire scientifique est bien plutôt mobilisé dans une perspective Nimby de manière fictionnelle, afin d'éviter l'aménagement. La volonté et les actes de protection de la biodiversité ne valent que s'ils restent en dehors du village et ne viennent pas empiéter sur la vie de ses habitants, leur vie quotidienne, la valeur foncière de leurs biens. Nul doute que s'il fallait faire passer un corridor écologique pour les animaux dans le village, des oppositions naîtraient tout autant chez les habitants.

Contrairement aux apparences, pour les opposants à la méthanisation, la mobilisation $\mathrm{du}$ discours scientifique écologique vient renforcer une conception et un rapport au paysage qui oscillent entre ce que Anaïs Belchun définit comme paysage-décor, paysage-émotion combinés en paysage-spectacle. "Quand ce modèle paysager est utilisé en relation avec un discours sur l'écologie, celui-ci se révèle souvent fondé sur une approche superficielle de l'écologie [...], le modèle du paysage-émotion perpétue une vision du monde naturaliste et anthropocentrée, valorisant les émotions et la grandeur de l'âme humaine face à une nature fantasmée [...]. En contradiction avec le message écologiste qu'il accompagne souvent, il perpétue une vision illusoire de la nature et une prise de distance entre l'homme et son environnement. » (Ibid.)

Le paysage émotion est au cœur des processus d'aménagement aujourd'hui, chez les opposants comme dans les services de l'État ou chez certains élus comme nous l'avons vu. Plus précisément, à partir des analyses d'Augustin Berque (2009), nous parlerons alors de la mobilisation d'une pensée paysagère qui participe du débat public, par distinction d'une véritable pensée du paysage. Cette pensée paysagère fait le lien entre les aménagements techniques-spatiaux et le paysage dans le sens où il y a bien un "souci du paysage », mais ce dernier n'est pas nommé, n'est pas évoqué comme un véritable enjeu. Caractéristique de la pensée paysagère (ibid.), l'intérêt pour le paysage est implicite et ne se retrouve pas dans le langage des protagonistes pour parler de la situation problématique. Une pensée paysagère qui se préoccupe du paysage en l'état et entend le préserver, en mettant en avant que toute introduction d'un nouvel élément conduirait à le dégrader. 


\section{BIBLIOGRAPHIE}

Abbott, A. « La pertinence actuelle de l'école de Chicago », dans Demazière, D. et Jouvenet, M., (dir.), Andrew Abbott et l'héritage de l'école de Chicago, Paris, Éditions EHESS, coll. « En Temps et Lieux », 2016.

Akrich, M., Callon, M., Latour, B. (dir.), Sociologie de la traduction, Paris, Presses des mines, coll. «Sciences sociales », 2006.

Akrich, M., "Les objets techniques et leurs utilisateurs ", dans Conein, B., Thévenot, L et Dodier, N. (dir.), Les objets dans l'action. De la maison au laboratoire, Paris, Éditions de l'EHESS, coll. «Raisons pratiques », 1993.

Béhar, L. et Simoulin, V., « Le Nimby (Not in My Backyard) : une dénonciation du localisme qui maintient l'illusion du local », Politiques et management public, vol 31/2, 2014, p. 151-167, mis en ligne en décembre 2016, URL : ttp ://journals.openedition.org/pmp/7000.

Belchun, A., « Les paysages de l'écologie : traversée critique des modèles paysagers, de la fiction verte à l'écopoétique ", Arts et Sciences, vol. 4, nº spécial, janvier 2020, URL : http:// www.openscience.fr/Les-paysages-de-l-ecologie-traversee-critique-des-modeles-paysagers-de-la Berque, A., Écoumène. Introduction à l'analyse des milieux humains, Paris, Belin, 2009.

Bourdin, S., Nadou, F., Raulin, F., « Les collectivités locales comme acteurs intermédiaires de la territorialisation de la transition énergétique : l'exemple de la méthanisation », Géographie, Économie, Société, vol. 21, 2019/4, p. 273-293.

Braud, P., Sociologie politique, Paris, LGDJ, 1998.

Callon, M., « Sociologie de l'acteur réseau », dans Akrich, M., Callon, M., Latour, B. (dir.), Sociologie de la traduction, Paris, Presses des mines, coll. « Sciences sociales », 2006.

Callon, M., et Law, J., «L'irruption des non-humains dans les sciences humaines : quelques leçons tirées de la sociologie des sciences et des techniques », dans Dupuy, J.-P. et Livet, P. (dir.), Les limites de la rationalité. Rationalité, éthique et cognition, t. 1, Paris, La Découverte, coll. « Recherches », 1997.

Cefaï, D., « Public, socialisation et politisation : Mead et Dewey », dans Cukier, A. et Debray, E. (dir.), La Théorie sociale de G. H. Mead, Lormont, Le Bord de l'eau, 2014.

Cefaï, D., « Vers une écologie des publics - Robert Ezra Park, l'opinion publique et le comportement collectif », dans Guth, S. (dir.), Modernité de Robert Ezra Park, Paris, L'Harmatan, 2008.

Chesneau, I. et Desplaces, G., « Les différentes mises en œuvre de la transition énergétique en milieu rural. Comparaison entre quatre régions ", dans Coste, A., D'Emilio, L. et Guillot, X., Ruralités post-carbone. Milieux, échelles et acteurs de la transition énergétique, Saint-Étienne, Publications de l'Université de Saint-Étienne, 2018, p. 178-185.

Descola, P., « Anthropologie de la nature », L'annuaire du Collège de France, $\mathrm{n}^{\circ} 112$, novembre 2013 , URL : http://journals.openedition.org/annuaire-cdf/737; DOI : https://doi.org/10.4000/annuairecdf.737

Descola, P., «Les formes du paysage ", cours au Collège de France, 2012, URL : https:// www.college-de-france.fr/site/philippe-descola/course-2011-2012.htm 
Descola, P., Par-delà nature et culture, Paris, Gallimard, coll. « Bibliothèque des sciences humaines ", 2005.

Goffman, E., La Mise en scène de la vie quotidienne, Paris, Éditions de Miniuit, coll. « Le sens commun », 1992.

Grafmeyer, Y, Joseph, I, L'école de Chicago. Naissance de l'écologie urbaine, Flammarion, coll. «Champs Essais », 2009.

Kortsch, T., Hildebrand, J., et Schweizer-Ries, P., « Acceptance of biomass plants-Results of a longitudinal study in the bioenergy-region Altmark », Renewable energy, vol. 83, p. 690-697.

Latour, B., « Défendre la nature : on baille. Défendre les territoires : on se bouge », Reporterre, le quotidien de l'écologie, (novembre 2017), URL : https://reporterre.net/Bruno-Latour-Defendre-lanature-on-baille-Defendre-les-territoires-on-se-bouge.

Latour, B., Nous n'avons jamais été modernes, Paris, La Découverte, 1991.

Lazzarotti, O.,(2015) «L'habiter sur un plateau », Annales de géographie, n 704, 2015/4, p. 335-337, URL : https://www.cairn.info/revue-annales-de-geographie-2015-4-page-335.htm

Le Breton D. (2012), L'interactionnisme symbolique, PUF, collection Quadrige.

Soulé, B., « Observation participante ou participation observante ? Usages et justifications de la notion de participation observante en sciences sociales ", Recherches Qualitatives, vol. 27, 2007, p. 127-140, URL : http://www.recherche-qualitative.qc.ca/documents/files/revue/ edition_reguliere/numero27(1)/soule.pdf.

Toublanc, M. et Bonin, S., « La fabrique politique et habitante des limites urbaines : études de cas à l'île de La Réunion ", Développement durable et territoires, vol. 10, $\mathrm{n}^{\circ} 2$, mis en ligne en juillet 2019, URL : http://journals.openedition.org/developpementdurable/14503; DOI : https://doi.org/ 10.4000/developpementdurable.14503

\section{NOTES}

1. La notion d'environnement mobilisée dans ce texte renvoie aux travaux de Philippe Descola (2013) : « [...] un territoire façonné par l'action humaine, [...] un substrat biophysique sur lequel se dépose en couches successives, l'histoire de sociétés singulières. »

2. Voire plus large pour ce qui est des réglementations internationales, qui peuvent être, en l'occurrence, européennes ou en partenariat avec des entreprises étrangères pour des aspects techniques d'exploitation.

3. Notons que la référence aux conflits liés à l'implantation d'éoliennes en milieu rural et la «défiguration» $d u$ paysage que les populations $\mathrm{y}$ associent sont également très souvent mobilisés comme arguments pour les opposants aux unités de méthanisation.

4. Au sens politique du terme.

5. Plantées soit avant, soit après la culture nouricière principale.

6. Le code de l'environnement défini les projets soumis à une évaluation environnementale, voir l'article R122-2 et Annexe à la section 1 du chapitre III du titre IX du livre V. 


\section{RÉSUMÉS}

Cet article propose d'analyser le paysage comme processus renvoyant à une action collective mobilisant différents groupes humains, quasi humains et non humains (Akrich et al., 2006), aux intérêts croisés, conflictuels ou convergents évoluant dans le temps, mais aussi, en relation de compétition, d'indifférence, d'accommodation, voire d'assimilation (Grafmeyer et Joseph, 2009). Une définition du paysage qui mobilise donc à la fois les théories de l'acteur-réseau, mais également l'approche sociologique interactionniste (Le Breton, 2012). Mobilisant une observation participante ethnographique réalisée à partir du rôle que nous avons acquis dans l'action collective elle-même, il s'agit d'analyser le processus d'implantation d'un projet d'aménagement participant de la production d'un paysage: l'installation d'une unité de méthanisation à 60 kilomètres de Paris. Le texte s'arrête ainsi sur l'omniprésence des registres environnementaux dans la structuration de ces situations en débat public et plus précisément sur la place du biodivers anthropisé comme cadre de cette situation. La notion de biodivers se construit à travers un prisme systématiquement anthropomorphique ou humano-référencé, par la mobilisation de la science à des fins d'argumentation. Elle intervient alors dans la constitution des publics (Cefaï, 2008 ; 2013) et de situations problématiques d'aménagement du territoire, qui implicitement relèvent d'une lutte pour le maintien en l'état d'un paysage.

This article proposes to analyse the landscape as a process relating to a collective action mobilising various human, quasi-human, and non-human groups (Akrich et al., 2006), with crossed, conflicting, and converging interests evolving over time, but who are also in situations of competition, indifference, accommodation, and even assimilation (Grafmeyer and Joseph, 2009). A definition of the landscape that therefore involves at the same time the actor-network theories and the sociological interactionist approach (Le Breton, 2012). Using ethnographic participative observation based on our role in the collective action, the aim is to analyse the process of the implementation of a development project contributing to the production of a landscape: the installation of a biogas plant $60 \mathrm{~km}$ from Paris. The article focuses on the omnipresence of environmental factors in the organisation of public debate and more precisely on the role of anthropized biodiversity as a framework. The notion of biodiversity is constructed through a systematically anthropomorphic or human-referenced perspective, through the mobilisation of science for the purposes of argumentation. It then intervenes in the constitution of audiences (Céfaï, 2015) and of problematic situations relating to land use planning, which are implicitly part of a struggle to maintain a landscape in its existing state.

\section{INDEX}

Mots-clés : action collective, situation problématique de l'aménagement, méthanisation, paysages acteurs-réseaux, biodiversité, biodivers, politiques publiques, aménagement $\mathrm{du}$ territoire

Keywords : collective action, problematic planning situation, mechanisation, actor-network landscapes, biodiversity, biodiverse, public policies, land use planning 


\section{AUTEURS}

\section{FABRICE RAFFIN}

Fabrice Raffin est maître de conférences en sociologie à l'université de Picardie Jules Verne, Laboratoire Habiter le Monde, Amiens.

fbraffin[at]gmail[dot]com

\section{CAMILLE DORMOY}

Camille Dormoy est doctorante en sociologie à l'université de Picardie Jules Verne, laboratoire Habiter le Monde. Sujet de sa thèse : «Gestion des déchets et propreté urbaine. Étude de cas de la ville d'Amiens - Somme ».

camille.dormoy[at]hotmail[dot]fr 